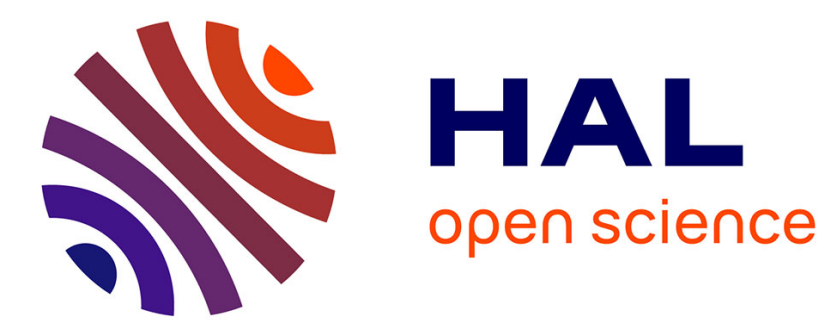

\title{
Air-filled soap bubbles for volumetric velocity measurements
}

\author{
Diogo C. Barros, Yanchong Duan, Daniel R Troolin, Ellen K Longmire
}

\section{To cite this version:}

Diogo C. Barros, Yanchong Duan, Daniel R Troolin, Ellen K Longmire. Air-filled soap bubbles for volumetric velocity measurements. Experiments in Fluids, 2021, 62 (2), 10.1007/s00348-021-03134-6 . hal-03202358

\section{HAL Id: hal-03202358 \\ https://hal-amu.archives-ouvertes.fr/hal-03202358}

Submitted on 19 Apr 2021

HAL is a multi-disciplinary open access archive for the deposit and dissemination of scientific research documents, whether they are published or not. The documents may come from teaching and research institutions in France or abroad, or from public or private research centers.
L'archive ouverte pluridisciplinaire HAL, est destinée au dépôt et à la diffusion de documents scientifiques de niveau recherche, publiés ou non, émanant des établissements d'enseignement et de recherche français ou étrangers, des laboratoires publics ou privés. 


\title{
Air-filled soap bubbles for volumetric velocity measurements
}

\author{
Diogo C. Barros · Yanchong Duan · Daniel Troolin · Ellen K. Longmire
}

Received: date / Accepted: date

\begin{abstract}
The use of air-filled soap bubbles for volumetric velocimetry in air flows is demonstrated experimentally. The tracers are produced by a novel system that seeds high number density soap bubble streams for particle image velocimetry applications. Particle number density considerations, spatial resolution and response time scales are discussed in light of current seeding techniques for volumetric measurements. The micro soap bubbles are employed to measure the 3D velocity field in intermediate size flow volumes of $75-500 \mathrm{~cm}^{3}$, which are difficult to measure with existing tracers such as liquid droplets or relatively large helium-filled soap bubbles, while resolving small scale flow variations. The mean volumetric concentration of approximately 50 tracers per $\mathrm{cm}^{3}$ provides a potential for highresolution measurements. Finally, the methodology is demonstrated for velocity measurements in the wake of a sphere immersed in a turbulent boundary layer. Both the wake structures and the boundary layer statistics are characterized successfully with a reasonable spatial resolution.
\end{abstract}

Keywords Soap bubbles · Particle Image Velocimetry

\section{Introduction}

Volumetric velocimetry is a key enabler for understanding turbulent flows, which are inherently unsteady and threedimensional. Multiple methods have been designed to mea-

Diogo C. Barros, Yanchong Duan and Ellen K. Longmire Department of Aerospace Engineering and Mechanics University of Minnesota, Minneapolis, MN 55455, USA

Daniel R. Troolin

TSI, Incorporated, 500 Cardigan Rd., Shoreview, MN, USA

E-mail: dtroolin@tsi.com

Diogo C. Barros

Aix Marseille Université, CNRS, IUSTI, Marseille, France

E-mail: diogo.camello-barros@univ-amu.fr sure 3D velocity fields accurately. In particular, particle image velocimetry (PIV) and particle tracking velocimetry (PTV) are now widely employed in various flow configurations (Discetti and Coletti, 2018). All of these techniques rely on imaging of discrete seeding tracers suspended in the flow. While larger seeding particles scatter more light from the illuminating source, smaller ones follow the flow more accurately and can resolve smaller scale variations. This trade off is inherent to particle-based velocimetry (Melling, 1997).

Adrian and Westerweel give a detailed discussion of possible tracer particles for gases and their performance (Adrian and Westerweel, 2011). Current seeding tracers for air flows include micron-size oil and di-ethyl-hexyl-sebacic acid-ester (DEHS) droplets (Kähler et al, 2002) as well as soap bubbles filled with a low-density gas such as helium. While droplets of size $1 \mu \mathrm{m}$ typically can follow fluid elements faithfully and remain suspended in the flow, the maximum size of the volumetric domain considered is usually limited to smaller than $50 \mathrm{~cm}^{3}$ (Scarano, 2013). On the other hand, large heliumfilled soap bubbles (HFSB) ranging from $1 \mathrm{~mm}$ to $5 \mathrm{~mm}$ allow only qualitative investigation of larger domains, as they do not faithfully follow the flow like neutrally buoyant bubbles (Kerho and Bragg, 1994). By contrast, sub millimeter $(300 \mu \mathrm{m})$ helium filled soap bubbles have been utilized successfully in both low and high-speed tomo-PIV configurations for large-scale aerodynamic applications (Scarano et al, 2015) in domains larger than $4500 \mathrm{~cm}^{3}$.

A compilation of volumetric measurements in air flows using these seeding strategies is shown in figure 1. There is a noticeable gap between volumetric domains in the range between nearly $50 \mathrm{~cm}^{3}$ and $5000 \mathrm{~cm}^{3}$. This is an effect of the large difference between the mean seeding diameters of DEHS droplets and HFSB bubbles. The purpose of the current work is to demonstrate the viability of smaller size airfilled soap bubbles as seeding tracers in gas volumes of intermediate size. The bubbles are generated by the rapid de- 
pressurization of a pressurized mixture of liquid and surfactant. The depressurization causes the gas to leave the liquid media. This controlled degasification inside a reservoir produces a large number of soap bubbles with mean diameter of $15 \mu \mathrm{m}$. The bubbles can be imaged easily either by low or high-speed light sources, yet they are small enough to resolve turbulent flow structures in intermediate-size domains.

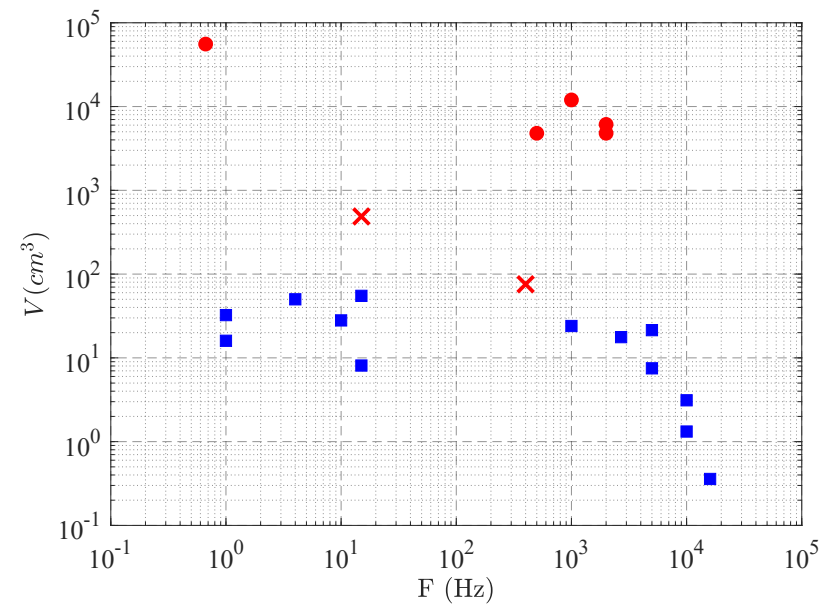

Fig. 1 Compilation of volumetric velocimetry investigations in air flows: acquisition frequency of the PIV set-up and the imaged volume. References: (Atkinson et al, 2011), (Kühn et al, 2011), (Cafiero et al, 2015),(Caridi et al, 2016), (Boushaki et al, 2017), (Pröbsting et al, 2013), (Ghaemi and Scarano, 2011), (Schneiders et al, 2016; Schneiders and Scarano, 2016), (Elsinga et al, 2006), (Schröder et al, 2008), (Humble et al, 2009), (Terra et al, 2017, 2018), (Scarano et al, 2015), (Violato et al, 2011), (Michaelis et al, 2012) and (Staack et al, 2010). The squares represent volumetric measurements using seeding particles with diameter of about $1 \mu \mathrm{m}$, while the circles indicate investigations where HFSB were used. The crosses represent the two volumetric domains considered in the present work

The paper is organized as follows. In section $\S 2$, we discuss spatial and image considerations for volumetric velocimetry in air flows, focusing on the seeding size. The methodology to generate the soap bubbles and their characteristics are outlined in $\S 3$, where a full description of the response time of the tracers is demonstrated theoretically and experimentally. A demonstration of the use of micro soap bubbles in volumetric wind-tunnel measurements is provided in section $\S 4$. Finally, our concluding remarks and perspectives on these new seeding tracers are discussed.

\section{Considerations of seeding size}

Consider a volume $V=L \times H \times W$ imaged by a camera with a pixel resolution $l^{*}$, pixel pitch $\Delta_{\text {pix }}$ equipped with an optical system with numerical aperture $f_{\#}$ by the use of an illuminating source with wavelength $\lambda$. In the volume $V, W$ represents the depth of the domain.
The depth of focus reads

$\Delta Z=4.88 f_{\#}^{2} \lambda\left(1+\frac{1}{M}\right)^{2}$.

Here, $M$ is the optical magnification $M=l^{*} \Delta_{\text {pix }} / L$.

Let $d_{p}$ be the diameter of the seeding particle. The particle image diameter can be calculated from

$d_{\tau}^{*}=\frac{\sqrt{\left(M d_{p}\right)^{2}+d_{\text {diff. }}{ }^{2}+d_{B}{ }^{2}}}{\Delta_{\text {pix }}}$,

where $d_{\text {diff. }}=2.44 \lambda f_{\#}(1+M)$ is the diffraction limited minimum image diameter and $d_{B}$ is the blur circle diameter (Raffel et al, 2018; Scarano, 2013).

Let us take the example of the experiments performed in Scarano et al (2015) with HFSB bubbles. The illuminated volume was such that $L=H=200 \mathrm{~mm}, W=120 \mathrm{~mm}, l^{*}=$ $1024 \mathrm{px}, \Delta_{\text {pix }}=20 \mu \mathrm{m}$ and $f_{\#}=32$ to ensure focus along all the depth $W(\Delta Z \sim 300 \mathrm{~mm})$. Assuming $d_{p}=400 \mu \mathrm{m}$ and neglecting $d_{B}$, the resulting particle image diameter is $d_{\tau}{ }^{*}=3 \mathrm{px}$, a consistent value found by the authors in their experiments of helium bubbles. Now, decreasing the domain size to $L=H=50 \mathrm{~mm}$ while keeping all other parameters the same, $d_{\tau}{ }^{*}=9 \mathrm{px}$ due to the change of optical magnification resulting in $\Delta Z \sim 30 \mathrm{~mm}$, i.e. an equivalent volume of approximately $V=75 \mathrm{~cm}^{3}$ when compared to $V=4800 \mathrm{~cm}^{3}$ from Scarano et al (2015). To obtain nearly optimal particle image diameters of $d_{\tau}{ }^{*}=3 \mathrm{px}$, the tracer size should be reduced to about $d_{p}=30 \mu \mathrm{m}$ or less considering the diffraction limit. Here, the value of three pixels is near optimal for minimizing both bias and random error in calculating PIV particle displacement (Adrian and Westerweel, 2011).

These examples clearly demonstrate that seeding tracers with diameter an order of magnitude smaller than HFSB are preferable for intermediate volume domains. At the same time, the tracers must be larger than DEHS droplets to scatter more light. Additionally, one should note that $d_{\tau}{ }^{*}=9 \mathrm{px}$ for a helium-filled soap bubble image would introduce a doublet pattern resulting from the presence of two glare spots (Scarano et al, 2015). This aspect makes the helium filled soap bubbles less suitable for such a field-of-view. Specifically, pairs of glare points add complexity and uncertainty to identification of particle centers in tracking methods and ambiguity in determination of the correct displacement peak in PIV correlation methods.

Another point of interest is the final resolution of the velocity measurements. Consider now the same domain $V=$ $L \times H \times W$ divided in voxels. We define $l_{\mathrm{IB}}$ as the length of the interrogation box (IB), i.e. the spatial resolution of the particle-based velocimetry. The number of seeding particles within the interrogation box is given by: 
$N_{p_{\mathrm{IB}}}=n_{v_{\mathrm{IB}}} \times C\left(\frac{\Delta_{\mathrm{pix}}}{M}\right)^{3}$.

Here, $n_{v_{\mathrm{IB}}}$ is the number of voxels per interrogation box, and $C=N_{p} / V$ is the volumetric particle concentration. The tracer concentration $C$ can be written as a function of the source density $N_{s}$ and the particle image diameter $d_{\tau}^{\star}$ (Scarano, 2013):

$C=\frac{N_{s}}{W}\left(\frac{M}{\Delta_{\text {pix }}}\right)^{2} \frac{4}{\pi d_{\tau}{ }^{* 2}}$.

Then, the number of particles per interrogation box reads

$N_{p_{\mathrm{IB}}}=n_{v_{\mathrm{IB}}} \frac{N_{s}}{W}\left(\frac{\Delta_{\mathrm{pix}}}{M}\right) \frac{4}{\pi d_{\tau}{ }^{* 2}}$.

Assuming $n_{v_{\mathrm{IB}}}=\left(l_{\mathrm{IB}} / l_{v}\right)^{3}$, where $l_{v}$ is the voxel length, and noting that $l_{v} M=\Delta_{\text {pix }}$, we obtain $l_{\mathrm{IB}} \propto d_{\tau}{ }^{\frac{2}{3}}$ for a desired number of particles per IB (usually between 5 and 10 particles) and optimal source density (Scarano, 2013). Thus, for intermediate sized volumes, a substantial gain in spatial resolution is expected by decreasing $d_{p}$ from $400 \mu \mathrm{m}$ to $30 \mu \mathrm{m}$, for example. The size of these tracers allows for a higher number density per unit volume which enables them to follow the turbulent structures of the flow and achieve higher spatial resolution measurements.

\section{Description of the micro-soap bubbles}

\subsection{Bubble generator}

Common methods to generate soap bubbles include pushing or blowing a gas stream through a liquid film. The support of the liquid film determines the dimension of the produced bubble. The method described here overcomes this limitation by using the process of cavitation in liquid media (Payri et al, 2009; Gharib and Kim, 2019). The mechanism governing the bubble generation is the rapid depressurization of a pressurized solution of liquid containing a certain amount of surfactant.

A schematic of the current device is seen in figure 2 . It consists of a 57 liter reservoir filled approximately onethird full with a 5\% surfactant-water solution. The surfactant used was a commonly available dish-washing detergent (Palmovile Pure+Clear) without any added dyes or fragrance and density of approximately $1.06 \mathrm{~g} / \mathrm{cm}^{3}$. The product ingredients were ammonium lauryl sulfate, lauramidopropylamine oxide, and isodeceth- 6 .

A tube at the bottom of the reservoir feeds the solution through a set of particulate filters followed by a reciprocating piston pump that pressurizes the fluid to approximately

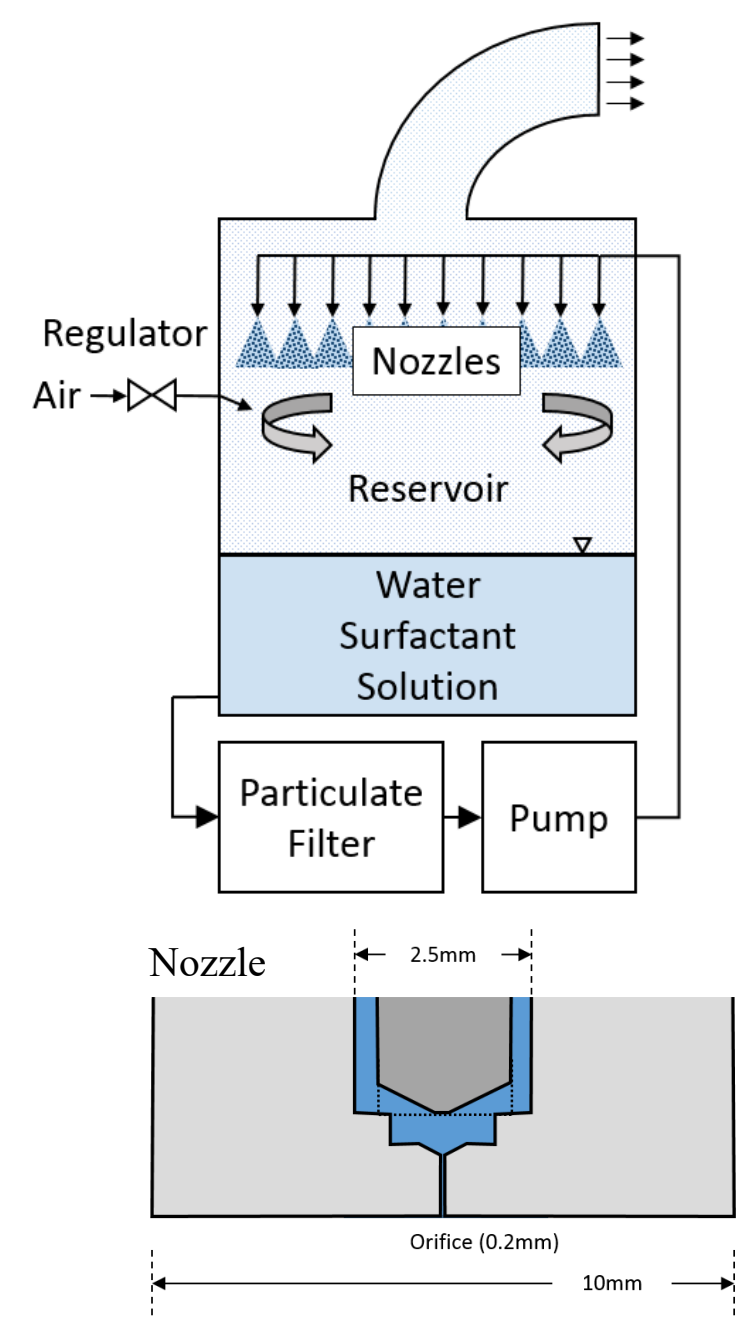

Fig. 2 Sketch of the bubble generator (top) and nozzle geometry (bottom).

70 bars. The pressurized liquid then travels through highpressure tubing to a set of 10 nozzles, each with outlet diameter of $0.2 \mathrm{~mm}$. Within each nozzle, the liquid is accelerated through an area ratio of 156:1 to decrease the local pressure. Gas volumes formed by cavitation then collapse and implode fragmenting into many small bubbles.

The bubble streams exiting the nozzles are directed downward toward the free surface of the reservoir bath. Pressurized air is introduced into the reservoir to generate a mild cyclonic flow that causes larger bubbles and droplets to impact the sidewalls and be reintroduced into the solution, while the remaining bubbles and the air stream exit through an opening at the top of the reservoir. Most of the bubbles created in this way remain suspended for long periods of time. 


\subsection{Bubble size characterization}

In order to characterize the bubble size, a phase Doppler particle analysis (PDPA) system was used (Bachalo, 1980). PDPA uses a set of crossed laser beams to create an interference fringe pattern at the point where the beams cross. Spherical particles passing through the beam crossing scatter light collected by a receiver with an array of three detectors. The phase lag of the scattered light between detectors is proportional to the particle diameter.

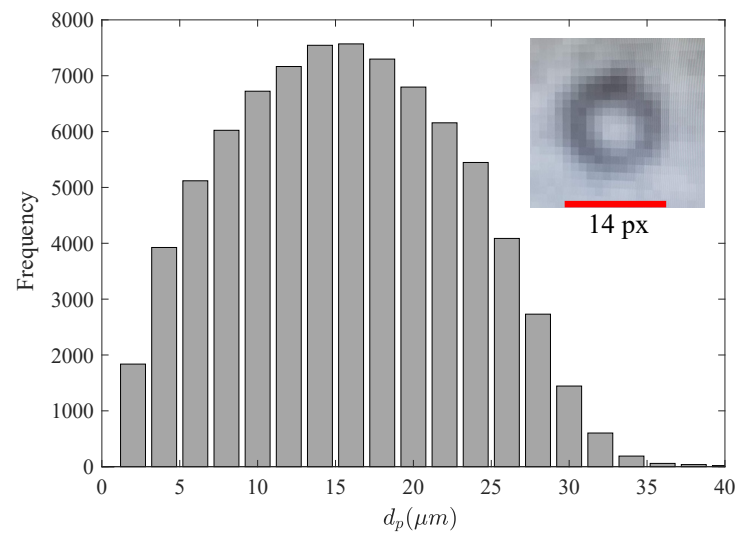

Fig. 3 Size distribution of the micro soap bubbles. The inset is a highmagnification shadowgraph image of a micro bubble with digital resolution of $1.5 \mu \mathrm{m} / \mathrm{px}$

A flexible tube $10 \mathrm{~cm}$ in diameter and 3 meters long was attached to the exit on top of the bubble generator. The PDPA system was positioned to measure the bubbles as they exited the tube. The generator produced a flux on the order of $10^{7}$ particles per second, resulting in a concentration of $10^{5}$ tracers $/ \mathrm{cm}^{3}$ at the outlet of the flexible tube. Figure 3 shows the measured diameter distribution of the bubbles generated by the device. The mean diameter was $14.7 \mu \mathrm{m}$ with a standard deviation of $7.3 \mu \mathrm{m}$. The inset shows a high-magnification shadowgraph image of a micro bubble obtained using a PowerView CCD $8 \mathrm{MP}$ camera $\left(\Delta_{\text {pix }}=\right.$ $5.5 \mu \mathrm{m}$ ) equipped with a long distance microscope Model K2 DistaMax yielding a digital resolution of $1.5 \mu \mathrm{m} / \mathrm{px}$.

\subsection{Theoretical estimation of the bubble response time}

A primary factor that affects the ability of the tracer to faithfully follow the flow is the sum of forces acting on the particle. A complete account for the motion of small particles in a given flow field can be found in Maxey and Riley (1983). Following Maxey and Riley (1983) and Mei (1996), Adrian and Westerweel (2011) proposed the following expression for the particle response time $\tau_{p}$ :

$\tau_{p}=d_{p}{ }^{2} \frac{\left|\rho_{p}-\rho_{f}\right|}{18 \mu \phi}$

where $\rho_{p}$ is the particle density, $\rho_{f}$ the fluid density and $\mu$ the dynamic viscosity of the ambient fluid. The factor $\phi$ corrects for the particle Reynolds number $\operatorname{Re}_{p}$ (see Clift et al (1978) for a detailed account). To estimate the response time of seeding tracers commonly used in airflow experiments, we compute $\tau_{p}$ using equation 6 and present the values in table 1 for various particles considering the Stokes regime $(\phi=1)$. We consider here air at $20^{\circ} \mathrm{C}$ and 1 atm. It is important to note that $\phi=1$ provides a conservative estimation of $\tau_{p}$ since the slip flow and resulting finite particle Reynolds number increases the value of $\phi$.

Micron-sized DEHS droplets are recognized to faithfully follow the flow due to their reduced size and can be considered as a reference particle. The characteristics of the DEHS particles used for the calculation of $\tau_{p}$ were the same as presented in Kähler et al (2002). The response time of DEHS particles obtained is about $3 \mu \mathrm{s}$, in very good agreement when compared to the experimental results of Ragni et al (2011), where $\tau_{p}=2 \mu s$ for shock accelerated tracers.

The same estimation was performed for Helium and air filled soap bubbles (HFSB and AFSB) with properties matching those listed in Faleiros et al (2018). Our calculation of $\tau_{p}$ using equation 6 for HFSB bubbles slightly lighter than air results in $\tau_{p}=90 \mu \mathrm{s}$. This lag is at least fifteen times smaller than the estimated response time of the AFSB bubbles, which have a much larger particle-fluid density difference. Measurements of HFSB response time performed by Faleiros et al $(2018,2019)$ in the flow upstream of a circular cylinder yielded experimental magnitudes of $\tau_{p}$ of $10-50 \mu s$ with a dispersion of approximately $40 \mu s$. They also reported a response time for AFSB of approximately $\tau_{p}=400 \mu \mathrm{s}$. In both cases, these experimental values of $\tau_{p}$ are smaller than our theoretical estimates. This can be explained by the significant slip velocity yielding a correction factor $\phi$ larger than unity. Meanwhile, in the absence of direct slip velocity measurements, equation 6 appears to be a good estimator of the considered response time. According to Kerho and Bragg (1994) and Afanasyev et al (2011), the wall-thickness of soap bubbles lies in the range 0.1-0.3 $\mu \mathrm{m}$. Indeed, from the characteristics of HFSB and AFSB tracers described above, a wall-thickness in the range 0.1-0.2 $\mu m$ was calculated to match the value of $\rho_{p}$ indicated by Faleiros et al (2018). Considering the bubble wall thickness in the range 0.1-0.3 $\mu \mathrm{m}$ and the particle diameter 10-30 $\mu \mathrm{m}$ , a response time range $20-160 \mu s$ was estimated for the micro soap bubbles of the current work, suggesting tracing fidelity capabilities comparable to the HFSB tracers. 
Table 1 Estimated response time for various tracers using equation 6.

\begin{tabular}{cccc} 
Seeding tracer & $\rho_{p}\left(\mathrm{~kg} . \mathrm{m}^{-3}\right)$ & $d_{p}(\mu m)$ & $\tau_{p}(\mu s)$ \\
\hline & & & \\
DEHS & 912 & 1 & 3 \\
HFSB & 1.1 & 550 & 90 \\
AFSB & 4.4 & 400 & 1590 \\
Current study & $20-170$ & $10-30$ & $20-160$ \\
\hline
\end{tabular}

\subsection{Measurement of the bubble response time}

The response time of the micro soap bubbles was obtained experimentally following the protocol described in previous investigations on HFSB bubbles (Scarano et al, 2015; Faleiros et al, 2018, 2019; Gibeau and Ghaemi, 2018; Gibeau et al, 2020). In steady flows, the particle response time can be computed as the ratio between the slip velocity and local fluid acceleration:

$\tau_{p}=\frac{\left|u-u_{p}\right|}{u_{p} \frac{\partial u_{p}}{\partial x}}$.

Here, $u$ is the fluid velocity, $u_{p}$ is the particle velocity and the local particle acceleration is kept the same as the fluid acceleration.

We consider the decelerating flow upstream of a circular cylinder. The experiments were performed in a closedcircuit wind-tunnel with test section dimension $1.3 \times 0.96 \mathrm{~m}^{2}$ and free-stream velocity $U_{\infty}=15 \mathrm{~ms}^{-1}$. The turbulence intensity at this free-stream condition is of order $1 \%$. A circular cylinder with diameter $D=70 \mathrm{~mm}$ and length $10 \mathrm{D}$ is mounted mid height, yielding a nominally two-dimensional flow at the cylinder midspan with a Reynolds number $R e=$ $\rho_{f} U_{\infty} D / \mu=6.8 \times 10^{4}$. This geometry gives a blockage ratio of $4 \%$ with negligible blockage effects (West and Apelt, 1982).

PIV measurements were performed to compute the fluid and particle (bubble) velocities $u$ and $u_{p}$ respectively. The local fluid velocity $u$ is approximated by the velocity field obtained from PIV measurements using oil droplets of diameter $\sim 1 \mu \mathrm{m}$ generated by a Laskin nozzle generator. This is justified by their very small response time and high tracing fidelity. An Evergreen $200 \mathrm{~mJ}$ laser was used to illuminate a planar field of view limited by $x \in[-80,0] \mathrm{mm}$ and $y \in[-30,30] \mathrm{mm}$, where $(x, y)=(0,0)$ is the stagnation point of the cylinder. A TSI Powerview 8MP camera $\left(3320 \times 2496\right.$ pixels, $\left.\Delta_{\text {pix }}=5.5 \mu \mathrm{m}\right)$ equipped with a 135 $\mathrm{mm}$ objective at numerical aperture $f_{\#}=5.6$ was used to acquire 3000 double-frame images with time separation of $\Delta \mathrm{t}=20 \mu \mathrm{s}$ and sampling rate $3 \mathrm{~Hz}$. The resulting image magnification is $M=0.165$. The images were processed using TSI INSIGHT4G, with a recursive, cross-correlation, ensemble PIV scheme with interrogation window of 64 pixels and $50 \%$ overlap yielding the final vector spacing of
$1.06 \mathrm{~mm}$. These parameters were used for both $u$ and $u_{p}$ measurements. Oil-droplet and bubble measurements were performed on separate days to avoid any cross-contamination of tracers.

The mean streamwise velocity and acceleration decay for bubbles $\left(u_{p}\right)$ along the stagnation streamline is depicted in figure 4 . The reference fluid velocity $u$ obtained from the oil droplets measurements is also displayed for comparison. The entire flow field (see inset) represents with fidelity the nominal potential flow upstream of the circular cylinder, and a similar flow topology was obtained using the reference oil droplets as seeding tracers. Along the streamline, the mean slip velocity $\left|u-u_{p}\right|$ is $0.12 \mathrm{~ms}^{-1}$, which corresponds to $0.8 \%$ of the freestream speed and $1.4 \%$ mean local velocity deviation.

To compute the bubble response time $\tau_{p}$, we first performed a local regression filter (loess in MATLAB) on both $u$ and $u_{p}$ to remove residual waviness of the data and to obtain a smooth velocity acceleration decay. Then, $\tau_{p}$ was calculated using equation 7 . The mean response time computed along the stagnation streamline in the region $0.5 D<x<0$ within a strip of $4 \mathrm{~mm}$ is $\tau_{p}=40 \mu \mathrm{s}$ with standard deviation $\sigma_{\tau_{p}}=30 \mu \mathrm{s}$. This selected region of large deceleration close to the stagnation point was also considered in Scarano et al (2015) and Gibeau and Ghaemi (2018) to obtain $\tau_{p}$ for HFSB bubbles. The measured $\tau_{p}=40 \mu \mathrm{s}$ lies within the theoretical range $20-160 \mu s$ estimated using equation 6 , where both bubble film thickness and diameter ranges were considered. A response time of $40 \mu \mathrm{s}$ would correspond to bubbles with diameter 15 (25) $\mu \mathrm{m}$ and film thickness of $0.15(0.1)$ $\mu m$, respectively. These values correspond well to the measured particle diameter distribution presented in $\S 3.2$.

It is important to consider that our calculation of $\tau_{p}$ and $\sigma_{\tau_{p}}$ considered velocity values obtained from a cross correlation algorithm within the PIV interrogation window, in which tracers with multiple properties exist. These effective time scales allows one to compute ideally minimum flow time scales to be measured by the current tracers. As discussed by Gibeau et al (2020), the resulting minimum measurable flow time scale reads $\tau_{f}=10 \times\left(\tau_{p}+2 \sigma_{\tau_{p}}\right)=$ $1000 \mu s$ with $95 \%$ confidence, accounting for a Stokes number $S_{k}=\tau_{p} / \tau_{f}<0.1$ for tracer reliability (Tropea and Yarin, 2007).

\section{Volumetric measurements}

Experiments were conducted in two wind-tunnels of the Department of Aerospace Engineering and Mechanics of the University of Minnesota to characterize the performance of the micro soap-bubbles at two volumetric scales. 


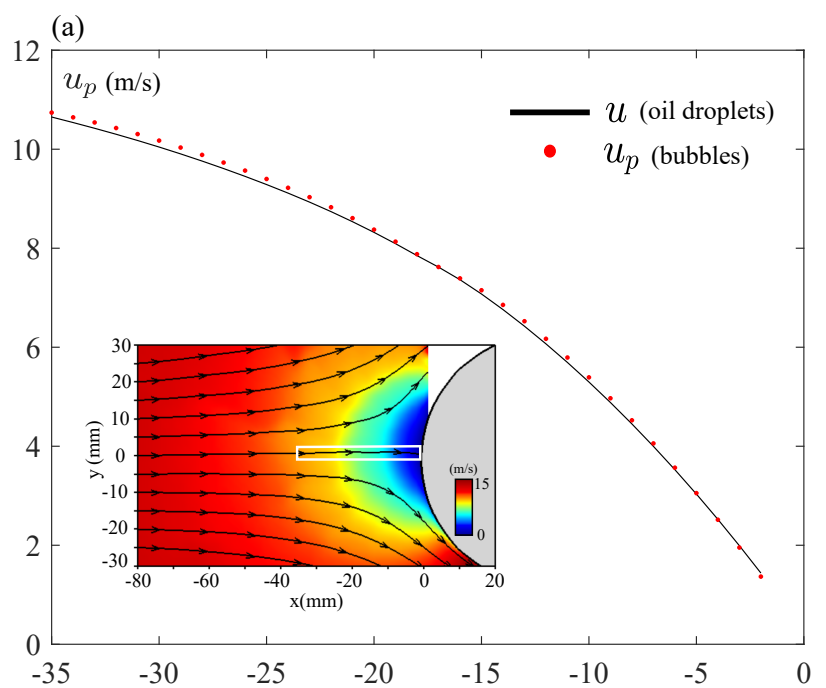

(b)

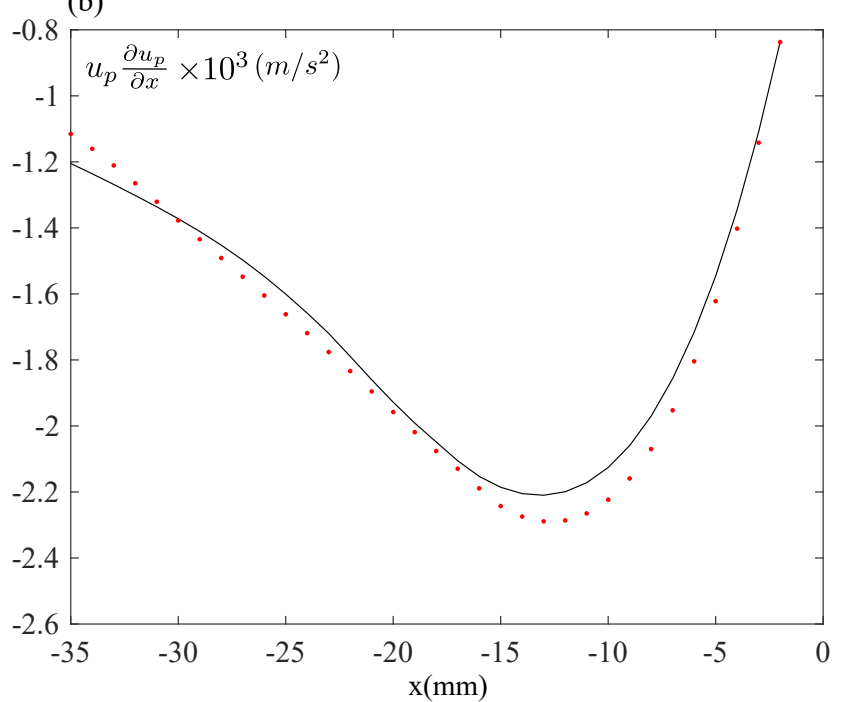

Fig. 4 Mean streamwise particle $\left(u_{p}\right)$ and fluid $(u)$ velocity (a) and acceleration (b) decay along the stagnation streamline. The velocity is represented by the colormap of $u_{p}$ and streamlines in the inset. The dashed rectangle indicates the region where the velocity and the acceleration were computed to calculate the response time.

\subsection{Bubble number density and robustness}

Experiments were performed in a large recirculating wind tunnel driven by a frequency-controlled $\mathrm{P}-38$ propeller (same as that used for the experiments in Section 3.4) in order to assess bubble quality in images and bubble robustness over different recirculation times. The flow conditioning upstream of the closed test section includes a honeycomb flow straightener with cell size of $8 \mathrm{~mm}$ followed by a set of four screens with openings of $700 \mu \mathrm{m}$. The contraction is threedimensional with a $6.5: 1$ area ratio. The test section measures $1.37 \mathrm{~m}$ wide, $0.96 \mathrm{~m}$ high, and $2.9 \mathrm{~m}$ in length. A volumetric imaging system consisting of four Phantom VEO340 $4 \mathrm{MP}$ cameras $\left(2560 \times 1600\right.$ pixels, $\left.\Delta_{\text {pix }}=10 \mu \mathrm{m}\right)$ fitted with $135 \mathrm{~mm}$ lenses and $f_{\#}=22$ operating at $800 \mathrm{~Hz}$ were aligned on the measuring volume illuminated by a $30 \mathrm{~mJ} /$ pulse dualhead Nd:YLF laser positioned on top of the wind tunnel, with a mirror directing the illumination down into the test section through a window on the top of the tunnel. Light volume optics were used to illuminate a volume of $490 \mathrm{~cm}^{3}$ with dimensions $130 \times 75 \times 50 \mathrm{~mm}^{3}$. This volume lies between the traditional volumetric domains investigated using either DEHS droplets or HSFB bubbles (see Figure 1). A synchronizer timing device with timing resolution of $0.2 \mathrm{~ns}$ coordinated the laser pulses and camera image capture, and the images of the bubbles were processed with INSIGHTV3V$4 \mathrm{G}$ software. Details of the processing routines used can be found in Boomsma and Troolin (2018).

The tests demonstrated the capability of performing largescale measurements with a free-stream flow velocity of $2.3 \mathrm{~ms}^{-1}$. The distribution of the tracers in the volume of interest is shown in figure 5. Here, 14000 bubbles were tracked, and the bubble digital image diameters were distributed across 3-4 pixels on average. After filling the tunnel with bubbles, the bubble generator was turned off, allowing the bubbles to recirculate for 5 minutes. During this period, there was a reduction of $32 \%$ in the number of tracers imaged. After an additional 5, 10, and 20 minutes, $64 \%, 47 \%$, and $33 \%$ of the initial number of bubbles remained, respectively. The measurements used to compute the response time in section 3.4 were performed 10 minutes after the generator was turned off.

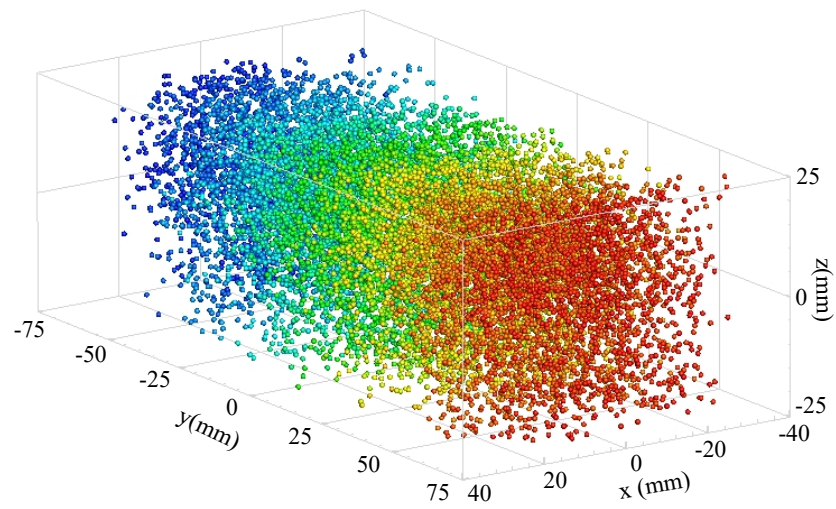

Fig. 5 Tracked particles in the large-scale volume $\left(490 \mathrm{~cm}^{3}\right)$. The particles are colored with their vertical $y$ coordinate to illustrate the seeding concentration in illuminated volume.

The initial relatively rapid decrease in concentration can be attributed to two potential factors. First, one may assume the existence of some droplets emanating from the bubble generator. Any droplets with diameter within the range 7-35 $\mu \mathrm{m}$ will tend to settle out in a period not exceeding 5 minutes after the bubble generator is turned off. In addition, water droplets in the range $1-4 \mu \mathrm{m}$ would tend to evaporate completely over the same period, while larger ones would decrease in diameter. Second, some fraction of micro soap 
bubbles (possibly the largest ones) may disappear in the first minutes due to impacts with surfaces and screens in the recirculating wind tunnel. The bubbles were also tested for larger free-stream speeds up to $25 \mathrm{~ms}^{-1}$. The bubbles were still robust, recirculating for long periods, although the concentration in the images was lower after a comparable time delay.

\subsection{Measurements in turbulent flow}

Another set of experiments was performed in an open-circuit wind-tunnel to assess the capability of bubbles to track relatively small scale motions in turbulence. Two flows were considered: a canonical turbulent boundary layer and the near wake downstream of a sphere embedded within the boundary layer.

\subsubsection{Experimental setup}

A sketch of the test section and experimental setup is shown in figure 6(a). The test section has a cross section of $600 \times$ $600 \mathrm{~mm}^{2}$ and a maximum flow speed of $40 \mathrm{~ms}^{-1}$. A trip wire was placed at the entrance of the test section to generate a turbulent boundary layer. The wind tunnel was driven by a frequency controlled variable speed motor and operated here with a fixed free-stream velocity $U_{o}$ of $3 \mathrm{~ms}^{-1}$. The estimated friction Reynolds number of the developing turbulent boundary layer at the measurement location was $R e_{\tau}=500$ with a viscous length scale $0.1 \mathrm{~mm}$.

For volumetric velocity measurements, a V3V-Flex traverse system from TSI Incorporated was employed and displaced according to the sketch of figures $6(a, b)$. Four 4MP cameras (Cam1 to Cam4, $2560 \times 1600$ pixels, $\Delta_{\text {pix }}=10 \mu \mathrm{m}$ ) equipped with Scheimpflug adapters were aligned in a planar configuration to image the illuminated volume. Each camera was equipped with a $135 \mathrm{~mm}$ lens with numerical aperture $f_{\#}=16$ and $f_{\#}=11$ for forward and back-scattering cameras, respectively. The acquisition frequency was 400 $\mathrm{Hz}$. The mean resulting optical magnification considering the four cameras was $M=0.28$. The measuring volume was illuminated by a $30 \mathrm{~mJ} /$ pulse dual-head Nd:YLF laser positioned at the open outlet of the wind tunnel. The volume of interest of $75 \mathrm{~cm}^{3}$ (VOI, dimensions $90 \times 56 \times 15 \mathrm{~mm}^{3}$ ) was located $1650 \mathrm{~mm}$ downstream of the trip wire with its side plane at the center line of the wind tunnel. A timing box was used to synchronize the laser pulses and the cameras. A sample raw image of the bubbles is depicted in figure 7. The bubbles display image diameters in the range $d_{\tau}{ }^{*}=1-4$ pixels.

Hereafter, the streamwise, wall-normal, and spanwise directions are denoted by $x, y$, and $z$, respectively. The instantaneous velocity fluctuations in the corresponding directions are denoted by $u, v$ and $w$, while the associated mean velocities are defined by $U, V$ and $W$, respectively. All quantities normalized by the boundary layer friction velocity $u_{\tau}$ and the kinematic viscosity $v$ are denoted with a superscript $(+)$. The root mean square value of a quantity is denoted by a prime $\left({ }^{\prime}\right)$ and its ensemble average is represented by an angle bracket $(\langle\rangle)$.

Measurements were conducted for two flow configurations: unperturbed and sphere-perturbed (SP) turbulent boundary layer. For the SP case, a sphere (diameter $D=25 \mathrm{~mm}$, $D^{+}=240$ ) held in place by a spanwise rod through its center was mounted inside the test section $109 \mathrm{~mm}$ upstream of the VOI center location (see red cross in figure 6(a)). Two SP cases were considered by changing the gap distance $G$ between the bottom of the sphere and the wall, $\mathrm{G}=0$ and $R$, where $R$ is the sphere radius. These two configurations are denoted as SP-G0 and SP-GR, respectively. In all cases, the time interval between successive frames was $150 \mu$ s and two sampling frequencies $60 \mathrm{~Hz}$ and $400 \mathrm{~Hz}$ were selected.

Both the calibration and image processing steps prior to volumetric velocity computation were performed in Insight V3V 4G. The 3D-particle tracking and reconstruction method is fully described in Boomsma and Troolin (2018). For image processing, the raw images were pre-processed using the following steps to reduce background and noise effects: 1 ) apply a $3 \times 3$ pixel Gaussian filter to remove the noise; 2$)$ subtract a local median $(9 \times 9$ pixels $)$ to remove the background; 3 ) subtract a constant intensity of 10 and multiply by a constant value of 5 to increase the contrast; 4) apply a $7 \times 7$ pixel Gaussian filter to facilitate the particle identification process. For each snapshot, the number of identified bubbles within the VOI was in the range 5000-8000.

For instantaneous velocity fields presented herein, the $3 \mathrm{D}$ velocity vectors associated with identified bubbles were binned into a uniform grid. Bad vectors (those exceeding the local mean value \pm three times the standard deviation in a given bin) were removed to obtain fields with a uniform vector spacing of $2 \times 2 \times 2 \mathrm{~mm}^{3}$. In the binned flow fields, any local outliers (exceeding the local mean value $\pm \beta$ times the standard deviation, where $\beta=3$ for $u, v$ and $w$ velocity components) were removed. Figure 8 shows a binned velocity vector field for one snapshot.

\subsubsection{Results}

The capability of the micro soap bubbles was first investigated under the unperturbed condition by comparing the resulting statistical velocity profiles with results from a direct numerical simulation (DNS) of a turbulent boundary layer at a similar Reynolds number $\left(R e_{\tau}=580\right)$. The numerical data set is extracted from Jiménez et al (2010). The experimental statistics were obtained based on ensemble averages of vectors present in 1500 snapshots. For the averaged statis- 
a)

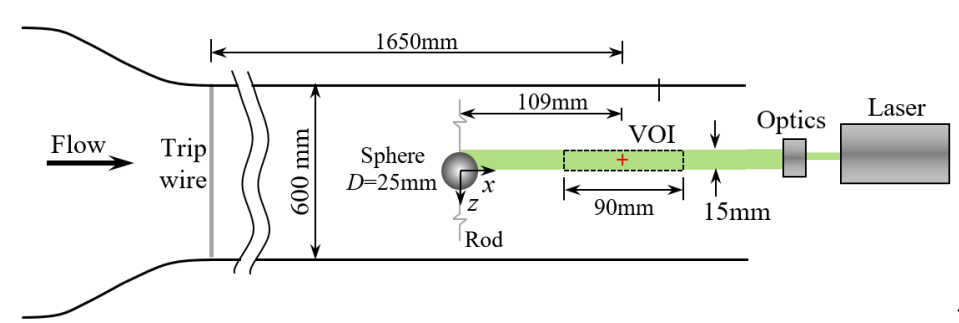

b)

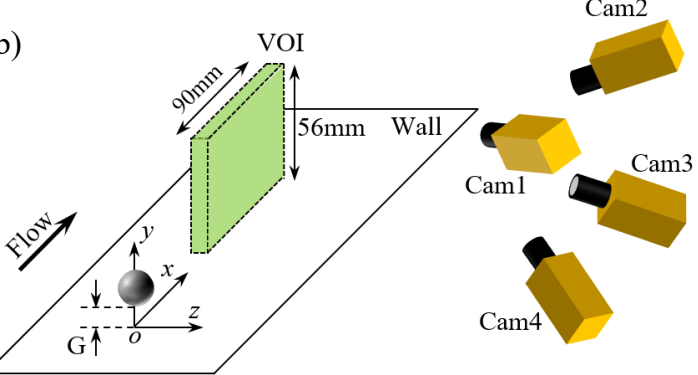

Fig. 6 Sketch of the experimental setup. (a) Top view of the wind tunnel; (b) 3D view of the coordinate system and arrangement of cameras, sphere, and VOI.

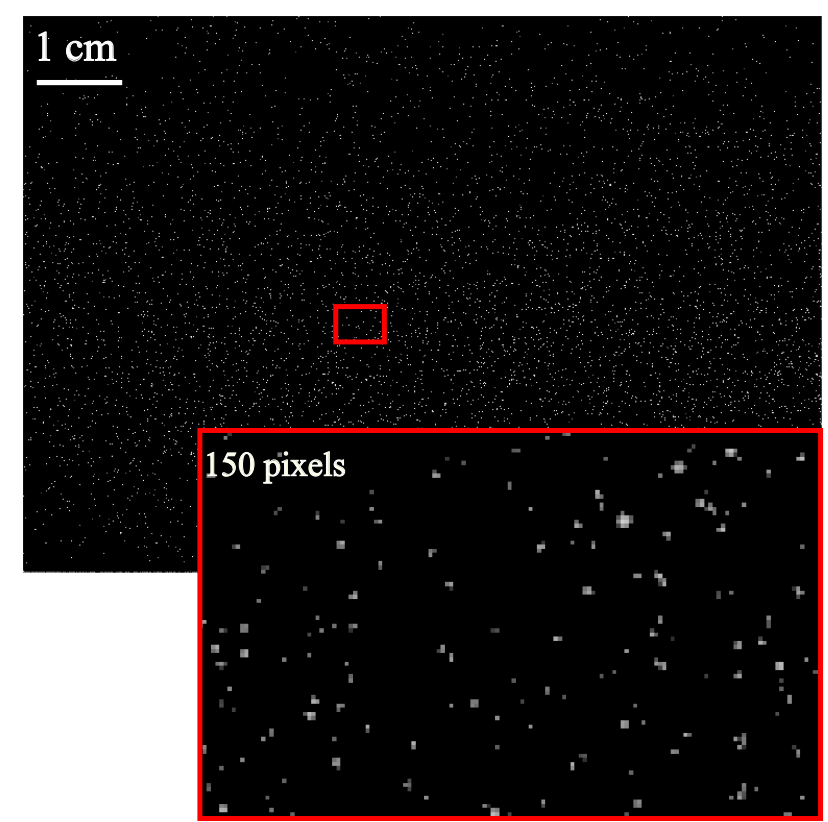

Fig. 7 A sample image of the bubbles acquired from one camera of the experimental set-up described in figure 6 . The inset field of view represents 150 pixels.

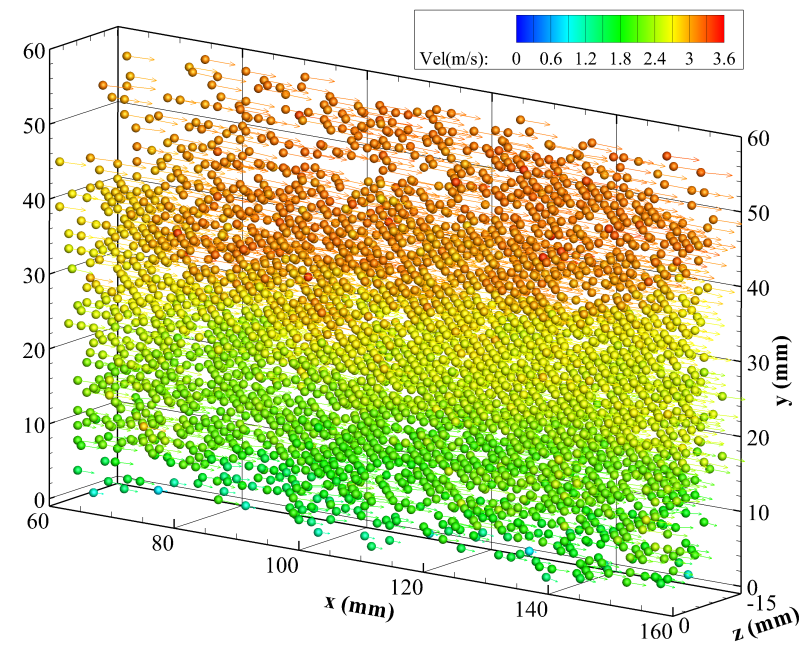

Fig. 8 Binned vectors in a uniform grid for the unperturbed case.
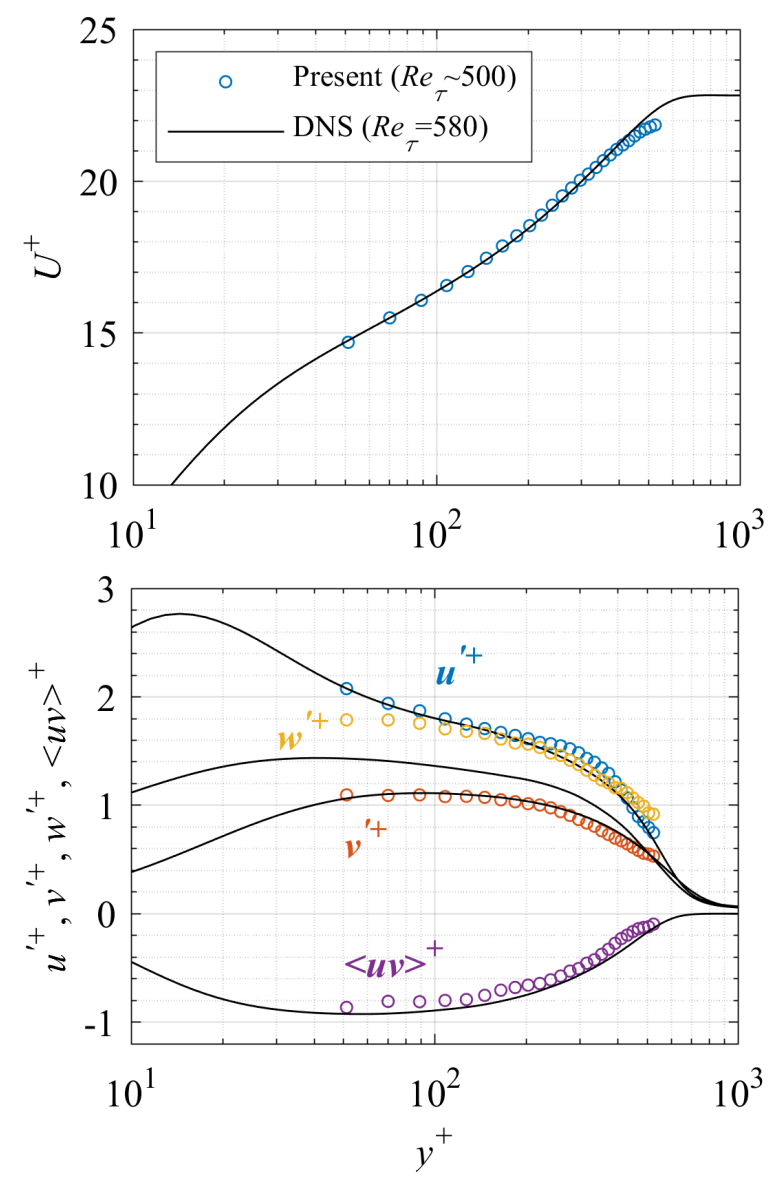

Fig. 9 Wall-normal velocity profiles of the inner scaled streamwise velocity (top) and turbulent intensities and Reynolds shear stress for the free-stream condition (bottom).

tics, all vectors were inserted into bins according to their location. Mean and rms values were computed at each location. Any outliers beyond three standard deviations in any component were removed, and the mean and rms values were recalculated. As presented in figure 9, the profiles of the mean streamwise velocity $U$, in-plane velocity fluctuations $\left(u^{\prime}, v^{\prime}\right)$ and the related Reynolds shear stress $\langle u v\rangle$ agree quite well with those from the DNS, thus demonstrating ex- 
cellent measurement quality. The values of spanwise velocity fluctuations $\left(w^{\prime}\right)$, however, are higher than in the DNS reference data reflecting the larger uncertainty in the out-ofplane component which is of order 0.5 pixels. Considering the inner length scale $l=v / u_{\tau}=0.1 \mathrm{~mm}$ obtained from the estimated friction velocity $u_{\tau}=0.142 \mathrm{~ms}^{-1}$, the associated flow time scale in the boundary layer is $\tau=l / u_{\tau} \sim 700 \mu \mathrm{s}$. Although this time scale is smaller than the measurable time scale estimated in section $3.4\left(\tau_{f}=1000 \mu \mathrm{s}\right)$, we note that the tracing fidelity of the employed bubbles is clearly sufficient to reproduce the Reynolds stresses as presented in figure 9.

Figure 10 depicts two flow snapshots each of the sphereperturbed configurations (SP-G0 and SP-GR). In the case where the sphere is close to the wall (SP-G0), the majority of vortical structures induced by the sphere are located within $y<D$. Also, these shed structures present mainly a negative vorticity sign (clockwise rotation), while alternating vortices are shed from the sphere for the SP-GR configuration shown in figures $10(\mathrm{~b}, \mathrm{~d})$. In this case, the vortical activity is spread across the region within $0.5 D<y<2 D$. The complexity of the vorticity field can be explained by the combination of vortices convected within the turbulent boundary layer, the ejected fluid below the sphere and the vortex shedding downstream of the sphere.

\section{Concluding remarks}

The current experiments demonstrate the feasibility of using air-filled micro soap bubbles to obtain high-quality planar and volumetric velocity measurements in air flows with intermediate-sized volumes in the range $75-500 \mathrm{~cm}^{3}$. This seeding methodology thus complements existing methods of $1 \mu \mathrm{m}$ liquid droplets optimized for smaller volumes and $400 \mu \mathrm{m}$ Helium-filled soap bubbles appropriate for larger volumes. The air-filled microbubble tracers, which have mean diameters of approximately $15 \mu \mathrm{m}$, offer much stronger scattering capability than $1 \mu \mathrm{m}$ droplets, yet also have relatively small response time. The measured mean response time was $40 \mu \mathrm{s}$ with a dispersion of $30 \mu \mathrm{s}$. The bubbles can be generated at high rates to fill large facilities or with relatively large local concentration ( 50 tracers per $\mathrm{cm}^{3}$ ) that can be sustained over time. When introduced into a recirculating wind-tunnel, the bubbles were robust and long lasting. When employed as tracers in an illuminated volume of a turbulent boundary layer, the bubbles yielded averaged mean and rms statistics of high quality. Also, instantaneous volumetric measurements allowed resolution and characterization of vortical structures in the wake of a sphere immersed in the same boundary layer. The capabilities of the seeding method described suggest that it is well suited for various additional particle tracking and time-resolved velocimetry techniques (Schanz et al, 2016).
Acknowledgements This work was supported by TSI Incorporated. This work was partially supported by the U.S. National Science Foundation (NSF CBET-1510154). The authors gratefully acknowledge the insightful comments from the three referees.

\section{References}

Adrian R, Westerweel J (2011) Particle image velocimetry. 30, Cambridge University Press

Afanasyev Y, Andrews G, Deacon C (2011) Measuring soap bubble thickness with color matching. American Journal of Physics 79(10):1079-1082

Atkinson C, Coudert S, Foucaut JM, Stanislas M, Soria J (2011) The accuracy of tomographic particle image velocimetry for measurements of a turbulent boundary layer. Experiments in Fluids 50(4):1031-1056

Bachalo WD (1980) Method for measuring the size and velocity of spheres by dual-beam light-scatter interferometry. Applied Optics 19(3):363-370

Boomsma A, Troolin D (2018) Time-resolved particle image identification and reconstruction for volumetric 4DPTV. In: 19th International Symposium on Applications of Laser Techniques to Fluid Mechanics, Lisbon, Portugal

Boushaki T, Koched A, Mansouri Z, Lespinasse F (2017) Volumetric velocity measurements (V3V) on turbulent swirling flows. Flow Measurement and Instrumentation 54:46-55

Cafiero G, Discetti S, Astarita T (2015) Flow field topology of submerged jets with fractal generated turbulence. Physics of Fluids 27(11):115,103

Caridi GCA, Ragni D, Sciacchitano A, Scarano F (2016) Hfsb-seeding for large-scale tomographic PIV in wind tunnels. Experiments in Fluids 57(12):190

Clift R, Grace J, Weber M (1978) Bubbles, drops, and particles. Academic Press, New York

Discetti S, Coletti F (2018) Volumetric velocimetry for fluid flows. Measurement Science and Technology 29(4):042,001

Elsinga GE, Scarano F, Wieneke B, van Oudheusden BW (2006) Tomographic particle image velocimetry. Experiments in Fluids 41(6):933-947

Faleiros D, Tuinstra M, Sciacchitano A, Scarano F (2018) Helium-filled soap bubbles tracing fidelity in wallbounded turbulence. Experiments in Fluids 59(3):56

Faleiros D, Tuinstra M, Sciacchitano A, Scarano F (2019) Generation and control of helium-filled soap bubbles for PIV. Experiments in Fluids 60(3):40

Ghaemi S, Scarano F (2011) Counter-hairpin vortices in the turbulent wake of a sharp trailing edge. Journal of Fluid Mechanics 689:317-356

Gharib M, Kim D (2019) Method and apparatus for the production of microscale bubbles by depressurization cavitation. US Patent App. 16/134,136 

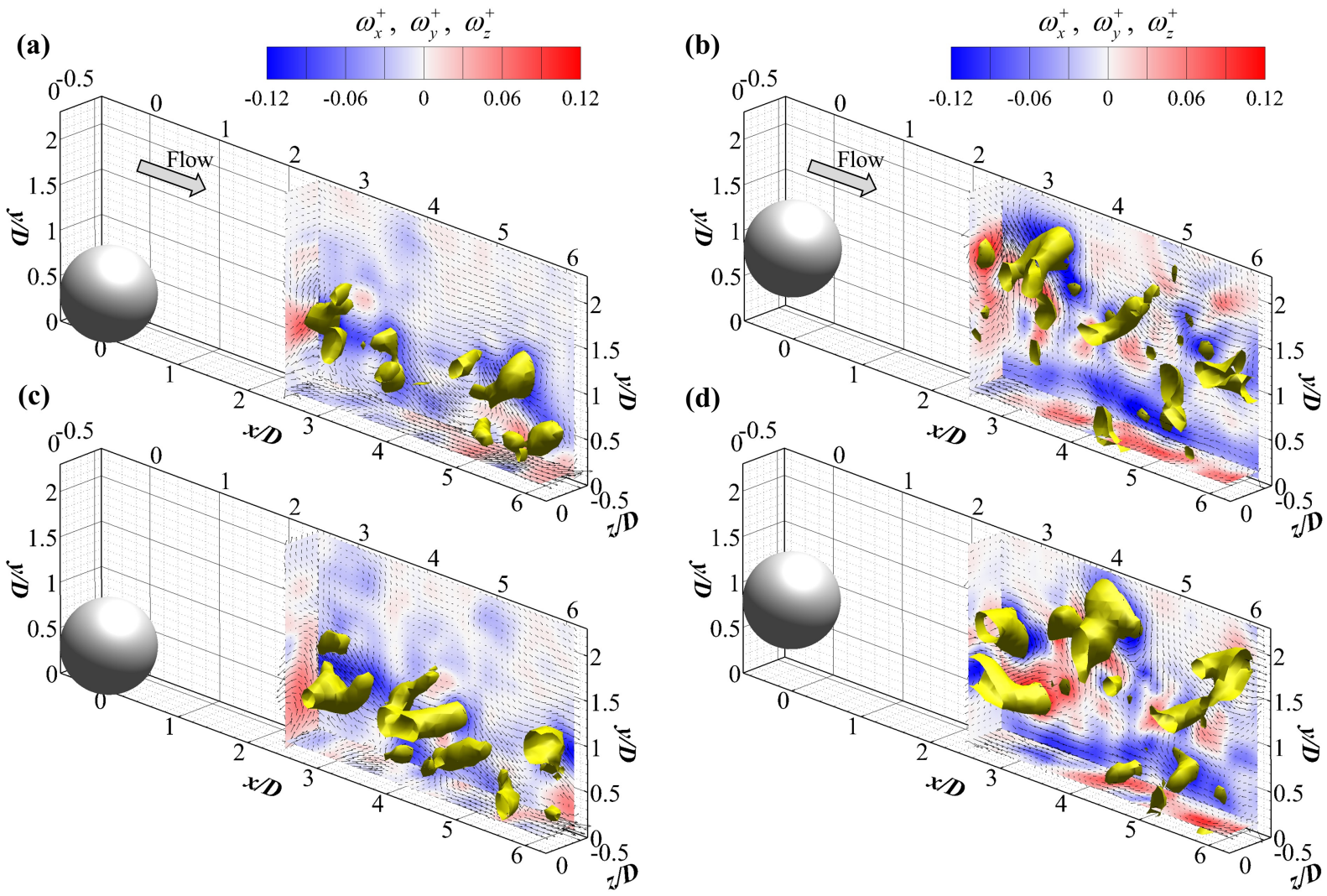

Fig. 10 Instantaneous flow fields for sphere-perturbed configurations. (a,c): SP-G0; (b,d): SP-GR. The top and bottom rows are two instants with time separation $\Delta t^{+}=13$. The vectors on each plane are the in-plane fluctuating velocities while the color denotes the out of plane component of vorticity. The yellow iso-surface depicts flow structures identified with the $Q$-criterion where $Q^{+}=0.001$.

Gibeau B, Ghaemi S (2018) A modular, 3D-printed heliumfilled soap bubble generator for large-scale volumetric flow measurements. Experiments in Fluids 59(12):178

Gibeau B, Gingras D, Ghaemi S (2020) Evaluation of a fullscale helium-filled soap bubble generator. Experiments in Fluids 61(2):1-18

Humble R, Elsinga G, Scarano F, Van Oudheusden B (2009) Three-dimensional instantaneous structure of a shock wave/turbulent boundary layer interaction. Journal of Fluid Mechanics 622:33-62

Jiménez J, Hoyas S, Simens MP, Mizuno Y (2010) Turbulent boundary layers and channels at moderate reynolds numbers. Journal of Fluid Mechanics 657:335-360

Kähler C, Sammler B, Kompenhans J (2002) Generation and control of tracer particles for optical flow investigations in air. Experiments in Fluids 33:736-742

Kerho MF, Bragg MB (1994) Neutrally buoyant bubbles used as flow tracers in air. Experiments in Fluids 16(6):393-400

Kühn M, Ehrenfried K, Bosbach J, Wagner C (2011) Large-scale tomographic particle image velocimetry using helium-filled soap bubbles. Experiments in Fluids 50(4):929-948
Maxey M, Riley J (1983) Equation of motion for a small rigid sphere in a nonuniform flow. The Physics of Fluids 26(4):883-889

Mei R (1996) Velocity fidelity of flow tracer particles. Experiments in fluids $22(1): 1-13$

Melling A (1997) Tracer particles and seeding for particle image velocimetry. Measurement Science and Technology 8(12): 1406

Michaelis D, Bomphrey R, Henningsson P, Hollis D (2012) Reconstructing the vortex skeleton of the desert locust using phase averaged POD approximations from time resolved thin volume tomographic PIV. In: 16th International Symposium on Applications of Laser Techniques to Fluid Mechanics, Lisbon, Portugal

Payri R, Salvador F, Gimeno J, De la Morena J (2009) Study of cavitation phenomena based on a technique for visualizing bubbles in a liquid pressurized chamber. International Journal of Heat and Fluid Flow 30(4):768-777

Pröbsting S, Scarano F, Bernardini M, Pirozzoli S (2013) On the estimation of wall pressure coherence using time-resolved tomographic PIV. Experiments in Fluids 54(7): 1567

Raffel M, Willert CE, Scarano F, Kähler CJ, Wereley ST, Kompenhans J (2018) Particle image velocimetry: a prac- 
tical guide. Springer

Ragni D, Schrijer F, Oudheusden BV, Scarano F (2011) Particle tracer response across shocks measured by piv. Experiments in fluids 50(1):53-64

Scarano F (2013) Tomographic PIV: principles and practice. Measurement Science and Technology 24(1):012,001

Scarano F, Ghaemi S, Caridi CG, Bosbach J, Dierksheide U, Sciacchitano A (2015) On the use of helium-filled soap bubbles for large-scale tomographic PIV in wind tunnel experiments. Experiments in Fluids 56(2):42

Schanz D, Gesemann S, Schröder A (2016) Shake-the-box: Lagrangian particle tracking at high particle image densities. Experiments in Fluids 57(5):70

Schneiders JF, Scarano F (2016) Dense velocity reconstruction from tomographic PTV with material derivatives. Experiments in Fluids 57(9):139

Schneiders JF, Caridi GC, Sciacchitano A, Scarano F (2016) Large-scale volumetric pressure from tomographic PTV with HFSB tracers. Experiments in Fluids 57(11):164

Schröder A, Geisler R, Elsinga GE, Scarano F, Dierksheide U (2008) Investigation of a turbulent spot and a tripped turbulent boundary layer flow using time-resolved tomographic PIV. Experiments in Fluids 44(2):305-316

Staack K, Geisler R, Schröder A, Michaelis D (2010) 3D$3 \mathrm{C}$-coherent structure measurements in a free turbulent jet. In: 15th International Symposium on Applications of Laser Techniques to Fluid Mechanics, Lisbon, Portugal

Terra W, Sciacchitano A, Scarano F (2017) Aerodynamic drag of a transiting sphere by large-scale tomographicPIV. Experiments in Fluids 58(7):83

Terra W, Sciacchitano A, Scarano F, van Oudheusden B (2018) Drag resolution of a PIV wake rake for transiting models. Experiments in Fluids 59(7):120

Tropea C, Yarin AL (2007) Springer handbook of experimental fluid mechanics, vol 1. Springer Science \& Business Media

Violato D, Moore P, Scarano F (2011) Lagrangian and eulerian pressure field evaluation of rod-airfoil flow from time-resolved tomographic PIV. Experiments in Fluids 50(4): 1057-1070

West G, Apelt C (1982) The effects of tunnel blockage and aspect ratio on the mean flow past a circular cylinder with reynolds numbers between 104 and 105 . Journal of Fluid Mechanics 114:361-377 